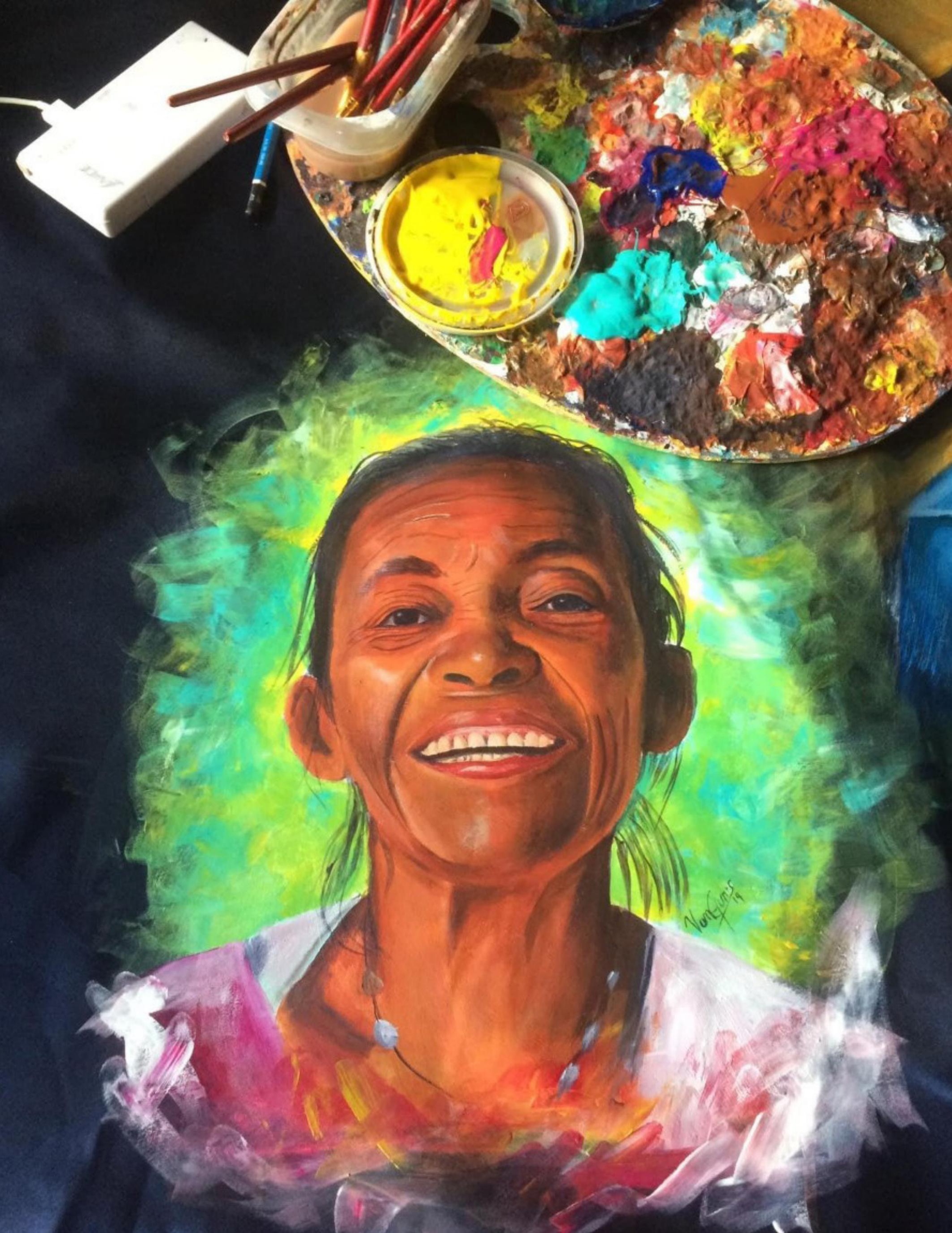




\section{MENSAJE DE JUAN PANADERO AL CONGRESO MUNDIAL POR LA PAZ}

Aquí estoy. Aquí ya estamos.

No tenemos cara. Somos

el planeta que habitamos.

Venid. No tenemos nombre.

Aunque todos respondamos

a una misma luz: el hombre. (...)

Matadnos. Nos mataréis.

Pero es más fuerte la vida

que la muerte que ofrecéis.

$Y$ al fin correréis la suerte

de los que matando llegan

a darle a su vida muerte. (...)

¿Queréis la guerra? No iremos.

Con la paz entre las manos

por arma, os enterraremos

¡Paz al mundo! Corazones

arrebatados y unidos de millones y millones.

Paz para toda la gente.

Se abran y cierren los ojos del día tranquilamente.
Paz en todos los hogares.

Paz en la tierra, en los cielos, bajo el mar, sobre los mares. Paz en la albura extendida del mantel, paz en la mesa sin ceño de la comida. En las aves, en las flores, en los peces, en los surcos abiertos de las labores.

Paz en la aurora, en el sueño. Paz en la pasión del grande y en la ilusión del pequeño. Paz sin fin, paz verdadera. Paz que al alba se levante y a la noche no se muera. ¡Paz, paz, paz! Paz luminosa.

Una vida de armonía sobre una tierra dichosa. Lo grita Juan Panadero. Juan en paz, un Juan sin guerra, un hombre del mundo entero. 


\title{
4. Cultura de paz y manejo del estrés a través de la estimulación neural
}

\author{
Francesca Randazzo Eisemann ${ }^{1}$ \\ Marisol Guerrero² \\ Alma Lorena Córdoba ${ }^{3}$ \\ DOI: https://doi.org/10.5377/pdac.v17i2.12740 \\ Recibido: 04/02/2021 - Aceptado: 20/05/2021
}

\begin{abstract}
Resumen: El propósito de este artículo es dar a conocer los resultados de una investigación cuali-cuantitativa que refleja la acogida de los talleres de la técnica de estimulación neural en la comunidad educativa de la Universidad Nacional Autónoma de Honduras (UNAH), impartidos para brindar a los participantes alternativas para enfrentar el impacto del estrés en sus vidas, y generar conocimientos para recomendar la implementación de esta técnica, como mecanismo accesible para responder a la escalada de la violencia y su repercusión en el ámbito educativo. La motivación para el desarrollo de los talleres se encuentra en el interés demostrado por los estudiantes en su mayoría de las carreras de Psicología, Medicina y Enfermería. Los hallazgos reflejan que las expectativas de las personas asistentes se centran, en primer lugar, en poder aprender o reforzar conocimientos sobre cómo mejorar su salud física, mental y emocional; y, en segundo lugar, comprender las emociones y los sentimientos de los demás. La violencia estructural se percibe sobre todo como falta de oportunidades, pobreza e injusticia. Los indicadores de estrés más frecuentes son preocupación excesiva, cansancio, falta de energía o concentración y ansiedad.
\end{abstract}

Palabras clave: meditación, educación, violencia, universidad, conflicto.

Peace culture and stress management through neural stimulation

Abstract: The purpose of this article is to present the results of the qualitative-quantitative research that reflects the reception of the workshops of the neural stimulation technique in the educational community of the National Autonomous University of Honduras (UNAH), given to provide participants with alternatives to face the impact of stress on their lives, as well as to generate knowledge to recommend the implementation of this technique, as an accessible mechanism to respond to the escalation of violence and its impact on the educational environment. The motivation for the development of the workshops is found in the interest shown by the students, mostly in the Careers of Psychology, Medicine and Nursing. The findings reflect that the expectations of the assistants are centered in first place, in being able to learn or reinforce knowledge about how to improve their physical, mental and emotional health; and second, to understand the emotions and feelings of others. Structural violence is perceived above all as a lack of opportunity, poverty and injustice. The most frequent indicators of stress are excessive worry, tiredness, lack of energy or concentration, and anxiety.

Key words: meditation, education, violence, university, conflict.

\section{Introducción}

El presente trabajo se centra en los hallazgos obtenidos de dos cuestionarios aplicados antes y después de una experiencia formativa concreta en Honduras a inicio de 2020, unas semanas antes de declararse la pandemia del COVID-19. Se trata de talleres de "Cultura de paz y manejo del estrés a través de la estimulación neural" (Merlo, 20 de febrero 2020; Comunicaciones UNAH-VS, 28 de febrero
2020), realizados en las ciudades de Tegucigalpa (20 y 21 de febrero de 2020), San Pedro Sula (24 y 25 de febrero de 2020) y Santa Rosa de Copán (26 y 27 de febrero de 2020). Estos talleres fueron impartidos por Manos Sin Fronteras Brasil, apoyados por Manos Sin Fronteras Honduras gracias a la gestión realizada por la Vicerrectoría de Relaciones Internacionales de Ciudad Universitaria-UNAH y el Departamento de Psicología de la Universidad Nacional Autónoma de Honduras del Valle de Sula.

1 Doctora en Sociología, Universidad de Santiago de Compostela. Profesora titular en la Universidad Nacional Autónoma de Honduras, e-mail: elfriede.randazzo@unah.edu.hn, ORCID ID: https://orcid.org/0000-0002-9572-0507

2 Enfermera, Máster en Sexología, Universidad de Alcalá de Henares. Secretaria de Manos Sin Fronteras Internacional, e-mail: marisolcomadrona@gmail.com

3 Máster en Psicología y Profesora titular en la Universidad Nacional Autónoma de Honduras. e-mail: alma.cordova@ unah.edu.hn 
El artículo plantea inicialmente un marco teórico y contextual que brinda un panorama sobre el flagelo de la violencia, pero también sobre las respuestas que la Cultura de Paz ofrece. En la metodología se abordan los aspectos relacionados con los instrumentos de medición e interpretación. Posteriormente se discuten los resultados recogidos sobre las carreras, el género, la edad y las expectativas de las personas interesadas en recibir el taller de estimulación neural; las percepciones sobre violencia estructural; los indicadores de estrés; y la respuesta de los talleres a las necesidades expresadas inicialmente.

\section{Metodología}

En este apartado se abordan aspectos metodológicos relativos a la elaboración de los instrumentos de medición y asimismo de interpretación. Como herramientas necesarias para construir una Cultura de Paz, éstos fueron diseñados en una perspectiva de investigación acción, es decir, no solo para extraer información sino también para resaltar aprendizajes o reforzar conocimientos sobre cómo mejorar la salud física, mental y emocional, equilibrar el propio sistema nervioso, resolver pacíficamente los conflictos, superar positivamente las tensiones, vivir con las demás personas en armonía, aprender a reconocer los intereses de los demás, y comprender las emociones y los sentimientos de los demás. Asimismo, y como uno de los resultados evidentes, se determina el interés demostrado hacia la estimulación neural según las carreras impartidas en la UNAH.

Se relacionan los métodos cualitativo y cuantitativo de forma simultánea, en especial en la etapa de interpretación, pues cualquier dato identificado implica tanto cualidad como cantidad. La investigación es por lo tanto cuali-cuantitativa, ya que la combinación de estrategias para el tratamiento de los datos se realiza en la obtención, organización, sistematización y análisis. Esto permite reducir la complejidad, riqueza y naturaleza de los datos y sus relaciones para lograr el comprenderlos (Mejía Arauz, 2003).

Con el fin de recopilar datos sobre la experiencia en base a lo planteado en la teoría, se realizó un cuestionario de entrada y otro de salida (retroalimentación), con preguntas cerradas y abiertas. Se trata de un estudio de caso en la Universidad Nacional Autónoma de Honduras, donde se registró, para el primer período del año 2020, una matrícula de 87,000 estudiantes a nivel nacional, de los cuales 55,000 fueron mujeres (63.2\%). La universidad es un espacio incluyente, que realiza esfuerzos para combatir las desigualdades, en especial las de género. De ellos 40,000 pertenecen a la Ciudad Universitaria Tegucigalpa; 16,000 a UNAH-VS San Pedro Sula y a otras filiales unos 2,000 estudiantes en cada una (Redacción web, 27 enero 2020), tal como la filial de Santa Rosa de Copán en donde se impartió también un taller. Es decir que, la población para este estudio fue de aproximadamente 58,000 estudiantes.

Para la elaboración de este artículo se hizo uso de los datos obtenidos y compilados a través de dos cuestionarios electrónicos con preguntas cerradas y abiertas. El primer cuestionario fue pasado durante la inscripción al Taller de Cultura de Paz y manejo del estrés a través de la estimulación neural, con una muestra de 611 inscripciones a los talleres de una población total de 58,000 estudiantes, lo que permite tener un nivel de confianza del 95\% para las respuestas. El segundo cuestionario se aplica como retroalimentación una vez culminados los talleres y la muestra es de 146 de un total 546 que asistieron a los talleres. La información cualitativa de este último instrumento se sistematiza a través de la Teoría Fundamentada (Glaser y Strauss, 1967).

\section{Marco teórico contextual}

\subsection{Violencia en Honduras}

Honduras, como El Salvador y Guatemala, está entre los países que tienen los índices de violencia y criminalidad en América Latina más altos (Banco Mundial, 2011), por lo que sus habitantes viven expuestos directa e indirectamente a daño físico, psicológico, social y económico, que repercute en las diferentes estructuras del país, sobre todo en la estructura familiar y escolar.

De acuerdo a diversas fuentes de investigación, uno de los grupos más afectados en el país es la juventud, en especial, entre las edades comprendidas de 12 a 30 años (Observatorio Nacional de la Violencia, 2015). Los centros educativos más vulnerables son aquellos donde acuden los niños y jóvenes de los barrios o colonias urbano-marginal de las principales ciudades del país. En general estas zonas se han visto afectadas por la presencia de maras, pandillas y el crimen organizado causantes de muertes violentas, extorsiones, restricciones de movimiento, amenazas, acoso sexual, venta de drogas y robos, entre otros, mermando la capacidad de acceso y permanencia en la escuela o colegio (UNICEF, 2018). De hecho, miembros de las bandas cri- 
minales realizan labores de reclutamiento, vigilancia e inteligencia; además 3 de cada 10 miembros de bandas criminales son menores de edad (Consejo Noruego para Refugiados, 2016).

Pero no sólo la violencia criminal y sus repercusiones están afectando a la población estudiantil, también la violencia entre pares está causando un impacto muy fuerte en la autoestima y seguridad física de los estudiantes, fenómeno no sólo observado en Honduras sino en todo el mundo. Estas formas de violencia son cotidianas y se viven en el interior de las escuelas y colegios. Se trata de prácticas como "burlas, amenazas, intimidaciones, riñas, apodos, insultos y toqueteos o manoseo que culminan con agresiones físicas, psicológicas y sexuales" (Ayestas, 2007, p. 18).

En relación a los estudiantes universitarios, se encuentran factores como la crisis intrafamiliar, la situación socioeconómica, la inseguridad ciudadana, específicamente la delincuencia callejera a la que se exponen al movilizarse desde o hacia el campus universitario, así como la relación con la docencia, las cuales tienen un impacto con profundos efectos negativos en la población estudiantil, según un estudio realizado en la Universidad Nacional Autónoma de Honduras en el Valle de Sula (Córdova, 2017). En éste se identificaron reacciones de estrés como preocupación excesiva, escasa confianza en sí, tristeza, miedo, irritabilidad, cansancio, entre otras, repercutiendo en la salud física y mental, y por tanto, en el rendimiento académico.

Todos estos indicadores de estrés frente a la violencia vivida por la población estudiantil, sea cual sea el nivel económico o social al que se pertenezca, pueden afectar a la salud mental y física de quien está expuesto directa o indirectamente (OPS, 2016). Fomentando o reforzando a nivel individual estrategias de afrontamiento, activas o adaptativas, se ayuda a enfrentar de manera positiva estos efectos negativos, bajando los niveles de ansiedad y fomentando la autoestima.

\subsection{Estrategias y respuestas desde la Cultura de Paz}

Entre las estrategias de afrontamiento, se encuentran las acciones de la organización Manos Sin Fronteras en diferentes países del mundo, especialmente a través de los centros educativos para llevar herramientas tales como la meditación como factor protector ante la violencia (Randazzo, 2020).
Manos Sin Fronteras Brasil lanzó en 2014 la campaña "5 Minutos Eu Medito", la cual promueve tanto la meditación, como la educación para la paz, la gestión de conflictos y la prevención de todo tipo de violencia, ya sea directa o estructural (MSF, 2017). Junto a la estimulación neural, técnica que aprovecha el potencial terapéutico de las manos, cuida del bienestar de las personas, promueve la prevención de enfermedades emocionales, el manejo del estrés y actúa en el tratamiento de trastornos como depresión, síndrome de pánico, ansiedad, entre muchos otros. Debido a su larga trayectoria nacional y experiencia internacional adquirida, cuenta con una rica experiencia en programas de formación, conociendo los que se derivan de la promoción de la Cultura de Paz y manejo del estrés, impartidos en módulos básicos y avanzados. Además, tienen un equipo de profesionales con amplia experiencia en facilitar la formación en grupos organizados (UNAH, 2020).

La campaña internacional "5 minutos, Yo medito" (Marano, 2014), tiene por lema "Si tú estás en paz, el mundo está en paz", y se difunde gracias a un App gratuita, que suma minutos por la Paz en el Mundo en un "meditómetro":

La campaña de "5 minutos, Yo medito" tiene como objetivo difundir la práctica de la meditación, de forma que llegue fácilmente a las nuevas generaciones. Para eso, nada mejor que el uso de una aplicación que además de los beneficios del ejercicio diario, suma los minutos meditados, generando una onda expansiva de equilibrio. Estar en las escuelas, universidades y empresas es una de las características de uso de esta aplicación. De esa manera, antes de cualquier actividad, las personas pueden usar la aplicación para meditar, generando un bienestar personal y colectivo (Eumedito, 2020).

La educación juega un rol fundamental en el proceso de construcción de paz y de manejo del conflicto (Smith, 2010). Es por ello que la Universidad Nacional Autónoma de Honduras firmó una Carta de Intención con la organización Manos Sin Fronteras Brasil (UNAH, 2020), en la que reconocen el interés común de unir fuerzas para lograr un vínculo efectivo entre los sectores público y privado, la sociedad civil y la academia, con el fin de lograr:

I. Formación Integral en Estudios de Paz que incluya, entre otras cosas, Investigación, Educación y Acción por la Paz, especialmente en las áreas de mediación y reconciliación 
en conflictos a partir de las necesidades que demandan los complejos procesos de dos transformaciones de una cultura de la violencia a una cultura de paz para contribuir a la solución de los principales problemas sociales, especialmente de los sectores más vulnerables de la sociedad.

II. La enseñanza de conocimientos socialmente útiles, que implica una constante actualización de contenidos del programa, incorporando nuevos temas como alternativa a la violencia y como respuesta creativa al conflicto.

III. Contribución a la cultura de paz y su acompañamiento mediante el establecimiento de un observatorio regional y local para generar información y mejorar las metodologías de investigación.

El fomento de la Cultura de Paz a través de habilidades para la vida es una propuesta de la Organización Panamericana para la Salud y la Organización Mundial para la Salud que está basada en la teoría de resiliencia y riesgo. El enfoque incluye las habilidades socio-cognitivas, la aptitud social y las habilidades de resolución de conflictos que sirven como mediadores de conductas. La resiliencia es esencial en el ámbito familiar y educativo para obtener éxitos y experiencias que determinan confianza y optimismo (Tuvilla, 2004). Además, en el caso de haber crecido en ambientes desfavorables, permite restituir la autoestima en la persona para volver a creer en sí misma y volver a desarrollar la capacidad de superación ante las adversidades.

La capacitación en torno a habilidades de vida implica identificar emociones, controlar la ira y otras emociones negativas; desarrollar el autocontrol y ampliar la comunicación y potenciar la habilidad de resolver problemas y planear el futuro, entre otras (Cardia, 2005). Estas son capacidades que se fortalecen y se desarrollan a través de la Estimulación neural una técnica promovida por la ONG internacional Manos Sin Fronteras, difundida desde hace más de 25 años y que se aplica en más de 35 países del mundo. Se basa en el ejercicio de meditación, la localización de los puntos vitales y la aplicación de la técnica. Cuenta con tratamientos para el equilibrio del sistema nervioso, con el fin de recuperar la serenidad, relajar el sistema nervioso y mantener el equilibrio psico-emocional en sólo 10 minutos, fortalece el sistema inmunológico y mantiene los diferentes elementos que circulan por la sangre en los niveles adecuados.
Manos Sin Fronteras es una organización sin fines de lucro sin vínculos políticos, filosóficos o religiosos: "Formada por personas de diferentes nacionalidades, creencias y formación que, después de experimentar los beneficios de estimulación neural, se han unido en el esfuerzo por compartir técnicas de bienestar, con la consciencia que el futuro de nuestra sociedad depende de cada uno de nosotros" (MSF, 2019).

"Nuestras manos son ilimitadas para ayudar a todos los seres humanos y al planeta", son palabras de la precursora de la técnica de estimulación neural, fundadora de Manos Sin Fronteras La Jardinera (2019), "nombre dado afectivamente porque ha dedicado su vida a sembrar la semilla de esta Enseñanza, que es capaz de salvar vidas" (MSF, 2019). En efecto la violencia, literalmente se cobra las vidas, especialmente las jóvenes (s.a. 21 de agosto del 2016; s.a., 16 de febrero del 2017).

La violencia no sólo lleva a la muerte, sino que son muchas sus consecuencias (Sánchez y Castillo, s.f.), algunas menos visibles pues repercuten en ámbito mental. En el marco de la proyección social de Manos Sin Fronteras en el mundo se desarrollan una serie de actividades destinadas a la promoción de la paz desde ámbitos muy diversos, incluso para disminuir la carga mental (Randazzo, Wan Stadnik, Costa y Bim, 2021). Es en ese marco que se realizan en Honduras, los talleres de "Cultura de paz y manejo del estrés a través de la estimulación neural" en los campus de Ciudad Universitaria de Tegucigalpa, UNAH-VS en San Pedro Sula y el Centro Universitario Regional del Occidente CUROC, en Santa Rosa de Copán, con la finalidad de mejorar la calidad de vida de los jóvenes universitarios, disminuir los niveles de estrés, mejorar la concentración y la oxigenación cerebral (Comunicaciones UNAH-VS, 28 de febrero 2020). Dichos talleres se diseñaron bajo la supervisión del Instituto de estimulación neural y terapias naturales, liderado por la Socióloga Lucía Peña (Instituto de Estimulación Neural y Terapias Naturales, s.f.), el cual se ocupa principalmente de la formación de masters, instructores y profesionales orientados a la difusión de la técnica (MSF, 2019).

Estudios cualitativos realizados en el ámbito educativo revelan que la meditación es una estrategia efectiva para combatir las inequidades reflejadas en la salud de las personas, al ayudar a mejorar la calidad de sueño, la alimentación y el ejercicio físico; asimismo hay tendencia de ser menos reactivos, con una mejor conciencia de sí y un mejor estado de ánimo (Bryan, Hamilton y Finn, 2018). 
La seguridad humana es un concepto pluridimensional que se refiere a un estado de bienestar integral, abarcando diversos elementos, entre ellos la seguridad física y la salud. Permite analizar las distintas maneras en las cuales las personas experimentan vulnerabilidad en su vida cotidiana. Asimismo, promueve la participación de los grupos socialmente vulnerables en la toma de decisiones y el control social para que el curso de acción se determine en gran medida localmente (OPS, s.f.). La seguridad humana impulsa la noción de un enfoque centrado en las personas, flexible, pues está basado en las percepciones y experiencias en cuanto a la vulnerabilidad y la multiplicidad de amenazas. Esto permite a las comunidades, por heterogéneas que sean, identificar sus necesidades específicas y elaborar respuestas adecuadas dirigidas a las realidades locales (Korc et. alt., 2016).

Dentro del marco de promoción de la autosuficiencia y la autodeterminación de las personas y las comunidades, y con el fin de sondear la percepción sobre la necesidad de herramientas de Cultura de paz, elementos de violencia estructural y síntomas de estrés, se utiliza una metodología cuantitativa y cualitativa de recolección en el contexto local.

La perspectiva de estudio es la Teoría de la resiliencia permite abordar grupos humanos que se encuentran en situaciones de riesgo, enfocándose en capacidades, posibilidades, fortalezas y adaptación saludable
(Cardia, 20005). Esta teoría se enmarca dentro de la Seguridad Humana (OPS, s.f.) y se complementa con el abanico de Habilidades para la vida, como camino para construir una Cultura de paz en el que se inserta la Estimulación neural.

\section{Discusión de resultados}

\subsection{Cuestionario de entrada}

Se aprovechó la inscripción a los talleres para pasar un cuestionario capaz de brindar información sobre las carreras interesadas en recibir taller de Estimulación neural, el género y edad, las expectativas del taller, las percepciones sobre violencia estructural y los indicadores de estrés.

\subsubsection{Carreras interesadas en recibir taller de Esti- mulación neural}

Las carreras que se muestran especialmente interesadas en recibir los talleres relacionados con conocimientos de estimulación neural (Cuadro $N^{\circ} 1$ ) son aquellas del área de Ciencias de la Salud y la Vida (53.5\%). Posteriormente se encuentran las áreas de Ciencias Sociales y Humanas (14.3\%), seguidas de Economía y Finanzas (9.5\%), y luego el resto de las carreras. Por lo tanto, se puede afirmar que existe un interés mostrado por el alumnado de todas las carreras, predominando en las de Psicología, Medicina y Enfermería (Gráfico №1).

\section{Cuadro $\mathrm{N}^{\circ} 1$ Carreras interesadas en recibir taller de estimulación neural}

\begin{tabular}{|c|c|c|c|}
\hline Carrera & Carreras agrupadas & Estudiantes & Porcentaje \\
\hline $\begin{array}{l}\text { Ciencias de la } \\
\text { Salud y la } \\
\text { Vida: }\end{array}$ & $\begin{array}{l}\text { Psicología, Medicina, Enfermería, Nutrición, } \\
\text { Odontología, Radiología, Química y Farmacia, } \\
\text { Biología, Microbiología. }\end{array}$ & 332 & $53.5 \%$ \\
\hline $\begin{array}{l}\text { Ciencias } \\
\text { Humanas y } \\
\text { Sociales: }\end{array}$ & $\begin{array}{l}\text { Derecho, Trabajo Social, Sociología, Desarrollo } \\
\text { Local, Lenguas, Pedagogía, Periodismo, Letras, } \\
\text { Historia, Antropología, Ecoturismo, Música, } \\
\text { Educación Física, Técnico en Alimentos. }\end{array}$ & 89 & $14.3 \%$ \\
\hline $\begin{array}{l}\text { Economía y } \\
\text { Finanzas }\end{array}$ & $\begin{array}{l}\text { Informática Administrativa, Contaduría Pública y } \\
\text { Finanzas, Microfinanzas, Administración de } \\
\text { Empresas, Banca y Finanzas, Economía, } \\
\text { Mercadotecnia, Administración Pública, Comercio } \\
\text { Internacional, Administración } \\
\text { Matemáticas. }\end{array}$ & 59 & $9.5 \%$ \\
\hline Otros $^{4}$ & & 131 & $22.7 \%$ \\
\hline Total & & 611 & $100 \%$ \\
\hline
\end{tabular}

Fuente: elaboración propia a partir de datos recopilados en las inscripciones para los talleres de Cultura de Paz y manejo del estrés en Honduras, en las ciudades de Tegucigalpa (20 y 21 de febrero de 2020) y San Pedro Sula (24 y 25 de febrero de 2020).

4 Es de notar que estos casos corresponden a carreras técnicas o especialidades diversas. 


\section{Gráfico №1 Carreras con mayor interés en la estimulación neural}

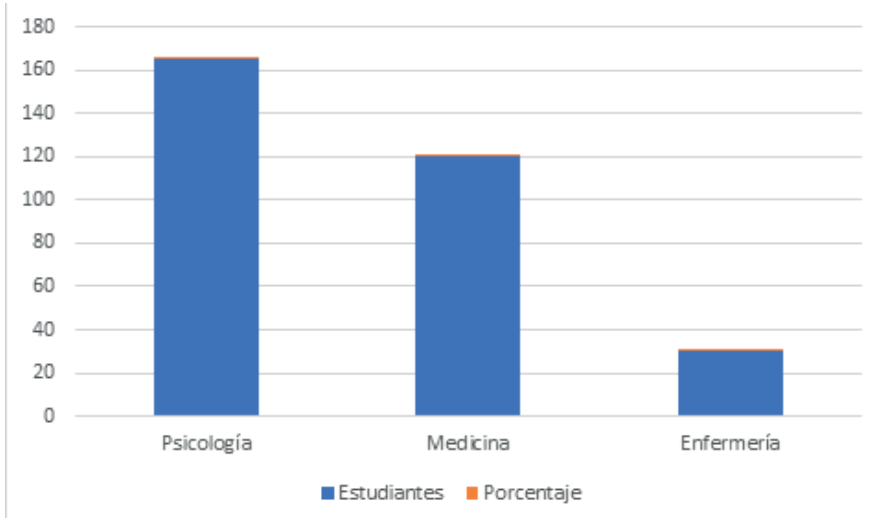

Fuente: Elaboración propia a partir de datos recopilados en las inscripciones para los talleres de Cultura de Paz y manejo del estrés en Honduras, en las ciudades de Tegucigalpa (20 y 21 de febrero de 2020) y San Pedro Sula (24 y 25 de febrero de 2020).

4.1.2. Género y edad de las personas interesadas en recibir taller de estimulación neural

El $76.2 \%$ son mujeres y el $23.8 \%$ hombres, es decir que hay tres veces más interés por parte de las mujeres que de los hombres en recibir los talleres de estimulación neural. Los rangos de edad son los siguientes: $0.8 \%$ menos de 18 años, $70.9 \%$ entre 18 y $29 ; 13.5 \%$ entre 30 y 39 y $14.8 \%$ más de 40 . Si bien, la mayoría corresponde a las edades promedio de asistencia a la universidad, esto muestra también un considerable interés por parte de la población joven.

\subsubsection{Expectativas del taller de estimulación neural}

Para las preguntas referidas a las expectativas sobre el taller (Cuadro $\mathrm{N}^{\circ} 2$ ) la muestra fue de 546, siendo el tamaño de la población 58,000 estudiantes. De las 546 respuestas, 362 respuestas corresponden a Tegucigalpa (65.9\%) y 184 a San Pedro Sula (34.1\%).

Las expectativas de las personas se centran primordialmente en aprender o reforzar conocimientos sobre cómo mejorar su salud, física, mental y emocional (84.7\%). En segundo lugar, comprender las emociones y los sentimientos de los demás $(58.6 \%)$, resolver positivamente los conflictos $(56.5 \%)$, superar positivamente las tensiones (56.3\%), equilibrar el sistema nervioso (53.2\%). Finalmente, también en porcentajes notables vivir con las demás personas en armonía (47\%) y aprender a reconocer los intereses de los demás (43.3\%). Todos estos aspectos son esenciales para construir una Cultura de Paz, y son referidos ya sea por la mayoría, o por porcentajes que se acercan mucho a ella.

El Cuadro $\mathrm{N}^{\circ} 3$ muestra las respuestas relacionadas con la violencia estructural, la cual se percibe sobre todo como falta de oportunidades (82\%), pobreza $(70.5 \%)$ e injusticia $(68.5 \%)$, reflejando las condiciones económicas y sociales del país. En segunda medida se percibe también como frustración (59\%), marginación (47.3\%), autoritarismo $(43.6 \%)$ y dominación y sumisión $(41.8 \%)$, evidenciando una dimensión relacional y de poder.

Cuadro №2 Expectativas de aprendizaje de los talleres de estimulación neural

\begin{tabular}{|l|c|}
\hline \multicolumn{1}{|c|}{ En el taller deseo aprender o reforzar conocimientos sobre... } & Porcentaje \\
\hline Cómo mejorar mi salud, física, mental y emocional & $84.7 \%$ \\
\hline Equilibrar mi sistema nervioso & $53.2 \%$ \\
\hline Resolver pacíficamente los conflictos & $56.5 \%$ \\
\hline Superar positivamente las tensiones & $56.3 \%$ \\
\hline Vivir con las demás personas en armonía & $47 \%$ \\
\hline Aprender a reconocer los intereses de los demás & $43.3 \%$ \\
\hline Comprender las emociones y los sentimientos de los demás & $58.6 \%$ \\
\hline
\end{tabular}

Fuente: elaboración propia a partir de datos recopilados en las inscripciones para los talleres de Cultura de Paz y manejo del estrés en Honduras, en las ciudades de Tegucigalpa (20 y 21 de febrero de 2020) y San Pedro Sula (24 y 25 de febrero de 2020). 


\section{Cuadro №3 Percepción sobre algunos indicadores de violencia estructural}

\begin{tabular}{|l|c|}
\hline \multicolumn{1}{|c|}{ En su entorno existe... } & Porcentaje \\
\hline Pobreza & $70.5 \%$ \\
\hline Injusticia & $68.5 \%$ \\
\hline Marginación & $47.3 \%$ \\
\hline Autoritarismo & $43.6 \%$ \\
\hline Frustración & $59 \%$ \\
\hline Falta de oportunidades & $82 \%$ \\
\hline Dominación y sumisión & $41.8 \%$ \\
\hline
\end{tabular}

Fuente: elaboración propia a partir de datos recopilados en las inscripciones para los talleres de Cultura de Paz y manejo del estrés en Honduras, en las ciudades de Tegucigalpa (20 y 21 de febrero de 2020) y San Pedro Sula (24 y 25 de febrero de 2020).

\section{Cuadro $N^{\circ} 4$ Percepción sobre algunos indicadores de estrés}

\begin{tabular}{|l|c|}
\hline \multicolumn{1}{|c|}{ Suele usted sentir o tener... } & Porcentaje \\
\hline Dolores de cabeza & $45.8 \%$ \\
\hline Cansancio & $65.2 \%$ \\
\hline Mala memoria & $47 \%$ \\
\hline Dolores y achaques frecuentes & $21.2 \%$ \\
\hline Falta de energía o concentración & $58.2 \%$ \\
\hline Rigidez muscular & $25.8 \%$ \\
\hline Diarrea o estreñimiento & $16 \%$ \\
\hline Consumo de comida en exceso & $25.6 \%$ \\
\hline Arrebatos de ira & $22.4 \%$ \\
\hline Problemas de sueño & $43.4 \%$ \\
\hline Problemas con alcohol u otras substancias & $4.2 \%$ \\
\hline Ansiedad & $52.6 \%$ \\
\hline Depresión & $22.8 \%$ \\
\hline
\end{tabular}

Fuente: elaboración propia a partir de datos recopilados en las inscripciones para los talleres de Cultura de Paz y manejo del estrés en Honduras, en las ciudades de Tegucigalpa (20 y 21 de febrero de 2020) y San Pedro Sula (24 y 25 de febrero de 2020).

\subsubsection{Indicadores de estrés}

En el Cuadro $\mathrm{N}^{\circ} 4$, vemos que los indicadores de estrés más frecuentes son cansancio $(65.2 \%)$, falta de energía o concentración (58.2\%) y ansiedad (52.6\%). En segundo lugar, se reporta mala memoria $(47 \%)$, dolores de cabeza (45.8\%) y problemas de sueño (43.4\%). En tercer lugar, se refiere consumo de comida en exceso (25.6\%), depresión $(22.8 \%)$, arrebatos de ira (22.4\%), dolores y achaques frecuentes $(21.2 \%)$, rigidez muscular (25.8\%), diarrea o estreñimiento (16\%) y problemas con el alcohol u otras substancias $(4.2 \%)$.

\subsection{El cuestionario de salida}

Una vez concluido el taller se solicitó llenar un cuestionario de salida con el fin de poder saber en qué medida se había respondido a las expectativas y necesidades expresadas inicialmente. En este cuestionario de retroalimentación, 58 de 146 personas, es decir casi el $40 \%$, calificaron el Taller de Cultura de Paz y manejo del estrés a través de la estimulación neural como "Excelente". Todas las respuestas fueron positivas, entre ellas las siguientes son denotativas: "Excelente para crecer"; "genial", "Me gustó mucho". Hubo muchos comentarios halagüeños como estos: "Los felicito por este taller, es algo novedoso y me parece interesante"; "Felicitaciones fue una experiencia muy enriquecedora".

Se recalca la importancia y la necesidad de los conocimientos brindados en el transcurso de las actividades: 
"Los talleres, son muy importantes ya que en cada uno que los hemos recibidos, nosotros, las personas, adquirimos nuevos conocimientos y por eso son actividades que deben estar siempre disponibles";

"se necesita implementar más estrategias de salud mental"; "en nuestro medio es necesario fortalecer estos temas".

Asimismo, se hace énfasis en los aprendizajes que fueron transmitidos durante los talleres:

"enriquece nuestros conocimientos, les agradecemos";

"Lo que aprendí lo estoy poniendo en práctica";

"Muy bonito y se aprende mucho";

"Quiero comprar libros relacionados al tema".

También se alude a la necesidad latente que existe de poder acceder a más formaciones de este tipo:

"es uno de los mejores que he asistido ojalá y sigan formando estos espacios";

"se deben fomentar más talleres sobre controlar los niveles de estrés con las técnicas de estimulación neural muy estimulante para trabajar la técnica y compartir los conocimientos adquiridos".

Muchos comentarios muestran que la gente se quedó con deseos de saber más y de seguir aprendiendo:

“¿Realizarán réplicas de este taller en otro momento?";

"quiero ser partícipe de otro curso así muy productivo";

"estoy muy interesada en participar";

"Me interesó bastante este curso, me gustaría recibir más cursos como este orientados a la importancia de la meditación".

"Necesito orientación para ordenar mi vida";

"Para poder adquirir más talleres como esté desearía me envíen un correo y así asistir"; "Aprendí mucho, y estoy poniendo en práctica lo aprendido, espero tomar más cursos como este";
"Me gustaría aprender más y poder ser algún día parte de los expositores e ir a impartir estas técnicas y taller en otras partes. Estuvo muy interesante";

"Me interesó bastante este curso, me encantaría recibir más, orientados a la importancia de la meditación".

De hecho, más de 100 personas (es decir más de dos tercios) pidieron en sus cuestionarios tener la oportunidad de tomar más talleres como el recibido.

Los talleres suscitaron muchas muestras de gratitud, entre las cuales cabe destacar las siguientes:

"Gracias por compartir excelentes talleres, es de mucha ayuda";

"Agradecerles su generosidad de compartir estos conocimientos que son tan beneficiosos"; "Gracias por el conocimiento y experiencias compartidas";

"Agradecer por tener estas iniciativas y poder compartirlas con la comunidad universitaria"; "Gracias por su paciencia al enseñar sobre el tema";

"Gracias por la oportunidad de participar"

\section{Conclusiones}

La teoría de resiliencia se aplica muy bien a grupos que se encuentran en situaciones de riesgo. Tiene un enfoque basado en capacidades, posibilidades, fortalezas y adaptación; así como en las condiciones que posibilitan un desarrollo más sano y positivo.

Con un alto grado de confianza se puede afirmar que entre la población estudiantil universitaria de Tegucigalpa y San Pedro Sula existe un interés demostrado en todas las carreras de asistir a los Talleres de Cultura de Paz y manejo del estrés a través de la estimulación neural, especialmente en las carreras como Psicología, Medicina y Enfermería. El alumnado percibe la violencia estructural sobre todo como falta de oportunidades, pobreza e injusticia. Los indicadores de estrés más frecuentes aludidos son cansancio, falta de energía o concentración, y ansiedad. Las expectativas de las personas se centran primordialmente en poder aprender o reforzar conocimientos sobre cómo mejorar su salud, física, mental y emocional $y$, en segundo lugar, comprender las emociones y los sentimientos de los demás. Estos aspectos 
son parte de los pilares fundamentales de la estimulación neural. Es decir que las expectativas se ven confirmadas en las respuestas positivas y muy alentadoras de la encuesta de salida.

Por lo tanto, se recomienda continuar con la implementación de la técnica de estimulación neural en los espacios universitarios y acompañarlos de instrumentos de medición que permitan evaluar sus impactos, especialmente para aumentar la resiliencia de poblaciones en condiciones de vulnerabilidad. Por tal razón los talleres en mención se encuentran dentro del enfoque preventivo y promocional de la Seguridad humana, al mejorar la capacidad de respuesta, reducir al mínimo las diferencias entre las personas e impulsar las sinergias entre los esfuerzos por proteger y empoderar a las comunidades.

\section{Referencias bibliográficas}

- Ayestas, M. N. (2007). Prevención de Violencia en Centros Educativos. Honduras. Recuperado el 21 de marzo del 2017 de: http://www.tzibalnaah. unah.edu.hn/bitstream/handle/123456789/254/ LB-00027. pdf? sequence $=2 \&$ isAllowed $=y$

- Banco Mundial (2011). Crimen y Violencia en Centroamérica. Un Desafío para el Desarrollo. LAC.

- $\quad$ Bryan, S.; Hamilton, M.; Finn, E. (2018). Mindfulness Meditation in College Students to Advance Health Equity. OBM Integrative and Complementary Medicine; 3(2):006; doi:10.21926/obm. icm.1802006.

- Cardia, N. (2005). Estado del arte de los programas de prevención de la violencia juvenil basados en la estrategia de la promoción del desarrollo de los jóvenes o que incorporan variables asociadas a este desarrollo. Washington, D.C.: Organización Panamericana de la Salud.

- Comunicaciones UNAH-VS (28 de febrero 2020). Realizan Taller de cultura de paz y manejo de estrés. Presencia. Recuperado el 20 de marzo de 2020 de: https://presencia.unah.edu. hn/noticias/nuevo-articulo-125/

- Consejo Noruego para Refugiados (2016). ¿Esconderse o huir? La situación humanitaria y la educación en Honduras.

- Córdova, A. (2017). Estrés en estudiantes de la Universidad Nacional Autónoma de Honduras en el Valle de Sula. Tesis de Maestría. UNAH.
San Pedro Sula, Honduras.

- Eumedito (2020). 5 minutos, yo medito. Recuperado el 18 de enero de 2020 de: http://www. eumedito.org/es/

- Glaser, B. y Strauss, A. (1967). The Discovery of Grounded Theory: Strategies for Qualitative Research. San Francisco, CA: Aldine.

- Instituto de Estimulación neural y terapias naturales (s.f.). Quiénes somos. Recuperado el 20 de marzo de 2020 de: http://institutoestimulacion. com/estimulacion-neural-2/manos-sin-fronteras/

- Korc, M.; Hubbard, S.; Suzuki, T.; y Jimba, M. (2016). Salud, resiliencia y seguridad humana: hacia la salud para todos. Japan Center for International Exchange and the Pan American Health Organization.

- La Jardinera (2019). Camino recto, camino de Luz. la-jardinera. Disponível em: https://www.facebook.com/notes/la-jardinera/camino-recto-camino-de-luz/2211026175641083/ Recuperado el 18 de abril de 2019.

- Marano (2014). 5 minutos, eu medito! Bandamaisbonita. Recuperado el 3 de marzo de 2020 de: http://bandamaisbonita.com.br/5-minutos-eu-medito/

- Mejía Arauz, Rebeca (2003) combinación estratégica: investigación sociocultural cualitativa-cuantitativa. Nómadas, No. 18, pp.20-27.

- Merlo, R. (20 de febrero 2020). Manos Sin Fronteras celebra taller para promover la cultura de paz. Presencia. Recuperado el 3 de marzo de 2020 de https://presencia.unah.edu.hn/noticias/ nuevo-articulo-116/

- MSF - Mãos sem Fronteiras (2017). Campanha 5 Minutos, eu medito. Mãos sem Fronteiras. Recuperado el 5 de septiembre de 2020 de https:// www.eumedito.org/quem-somos/.

- MSF- Manos sin Fronteras (2019) Recuperado el 5 de septiembre de 2020 de https://www. msfint.com/es/historia/

- Observatorio Nacional de la Violencia (2015). Boletín especial sobre homicidios de estudiantes. Edición Especial No. 29, Tegucigalpa, Honduras. Recuperado el 19 de noviembre de 2018 de: http://www.tzibalnaah.unah.edu.hn/ 
bitstream/handle/123456789/4771/BEP_Ed29. pdf? sequence $=2 \&$ isAllowed $=y$

- OPS - Organización Panamericana de la Salud (s.f.). Salud, Resilencia y Seguridad Humana. Hacia la Salud Para Todos. Recuperado el 6 de febrero de 2020 de: https://www.paho.org/hq/ index.php?option=com_content\&view=article\&i$d=11977$ :health-resilience-human-security\&Itemid=820\&lang=es

- OPS - Organización Panamericana de la Salud (2016). Informe sobre la situación mundial de la prevención de la violencia 2014. Washington, DC: OPS.

- Randazzo, F. (2020). La meditación en espacios educativos como factor protector ante la violencia. Pre-impresión. https://doi.org/10.5281/zenodo.4414879

- Randazzo Eisemann, F.; Wan Stadnik, A.; Costa de Miranda, A.; Bim, S. (2021) A Meditação guiada de La Jardinera para aliviar a carga mental das mulheres. Pre-impresión.

- Redacción web (27 enero 2020). UNAH abre las puertas a más 87 mil estudiantes en I PAC 2020. Tiempo digital. Recuperado el 6 de febrero de 2020 de: https://tiempo.hn/unah-abre-las-puertas-a-mas-87-mil-estudiantes-en-i-pac-2020/

- s.a. (21 de agosto del 2016). Jóvenes estudiantes afectados por ola de violencia. La Tribuna. Recuperado el 24 de marzo del 2017 de: http:// www.latribuna.hn/2016/08/21/jovenes-estudiantes-afectados-ola-violencia/

- s.a. (16 de febrero del 2017). Jóvenes son los más expuestos a la violencia. La Tribuna. Recuperado el 23 de marzo del 2017 de: http://www. latribuna.hn/2017/02/16/jovenes-los-mas-expuestos-la-violencia/

- Sánchez, A. y Castillo, D. (s.f.). Incidencia delictiva contra estudiantes de la UNAH. Universidad Nacional Autónoma de Honduras. Recuperado el 8 de enero de 2020 de: http://www.tzibalnaah. unah.edu.hn/bitstream/handle/123456789/286/ LB-00013.pdf?sequence $=2 \&$ isAllowed $=y$

- Smith, A. (2010). The influence of education on conflict and peace building. UNESCO/ Education for All Global Monitoring Report.
- Tuvilla, J. (2004). "Cultura de Paz y Educación" en Molina, Beatriz y Muñoz, Francisco. Manual de Paz y Conflictos. Granada: Eirene.

- UNAH - Universidad Nacional Autónoma de Honduras. (2020). Carta de Intención entre Manos sin Fronteras y la Universidad Nacional Autónoma De Honduras (UNAH).

- UNICEF (2018). Una lección diaria. Acabar con la violencia en las escuelas. Nueva York: Fondo de las Naciones Unidas para la infancia. División de Programas/Protección de la Infancia. 